\title{
Parenting stress and affective symptoms in parents of autistic children
}

\author{
GONG Yun ${ }^{1}$, DU YaSong ${ }^{1 *}$, LI HuiLin ${ }^{1}$, ZHANG XiYan ${ }^{1}$, AN Yu ${ }^{2} \&$ WU Bai-Lin ${ }^{2}$ \\ ${ }^{1}$ Shanghai Mental Health Center, Shanghai Jiao Tong University School of Medicine, Shanghai 200030, China; \\ ${ }^{2}$ Institutes of Biomedical Sciences, Fudan University, Shanghai 200032, China
}

\begin{abstract}
We examined parenting stress and mental health status in parents of autistic children and assessed factors associated with such stress. Participants were parents of 188 autistic children diagnosed with DSM-IV criteria and parents of 144 normally developing children. Parents of autistic children reported higher levels of stress, depression, and anxiety than parents of normally developing children. Mothers of autistic children had a higher risk of depression and anxiety than that did parents of normally developing children. Mothers compared to fathers of autistic children were more vulnerable to depression. Age, behavior problems of autistic children, and mothers' anxiety were significantly associated with parenting stress.
\end{abstract}

autistic children, parenting stress, mental health, parenting stress index

Citation: Gong Y, Du YS, Li HL, Zhang XY, An Y, Wu BL. Parenting stress and affective symptoms in parents of autistic children. Sci China Life Sci, 2015, 58: 1036-1043, doi: 10.1007/s11427-012-4293-Z

Childhood autism is a subtype of pervasive developmental disorders characterized by severe deficits in social interaction and communication, handicaps in verbal and nonverbal communication, an extremely limited range of activities and interests, and stereotypic and repetitive behavior. Most children also have accompanying intellectual disability and abnormal feelings. Generally, children present with symptoms of autism before three years of age [1]. The prevalence of autism is 13 per 10000 , with the male-female ratio varying from 1.33 to 16 , and a mean male-female ratio of $4: 1$ [2]. The prognosis for children with autism is poor. In addition, there is no effective treatment outside of special education. Abnormal behavior problems and deficits in language and social communication cause great stress to parents.

Parenting stress is an adverse psychological reaction to the demands of being a parent [3]. In the past 10 years, most research on autism in China has focused on children with this disorder while neglecting parents' stress and mental

*Corresponding author (email: yasongdu@yahoo.com.cn) health. Parenting a child with developmental disabilities is challenging. These parents experience higher levels of stress compared to parents of normally developing children [4-7]. Parenting stress related to raising an autistic child is significantly greater than that experienced by parents who have children with other developmental disabilities, such as Down syndrome, or parents with normally developing children because of severe impairments in language, social interaction, and self-care ability in autistic children $[1,4,6,8]$.

The symptoms and poor adaptability of autistic children require parents to spend more energy in the course of raising their child. Changes in mood are more common in these parents than in parents of normally developing children. For example, they may often feel tired and depressed. Hamlyn-Wright [9] showed that parents of children with autism reported higher levels of depression and anxiety than parents of children with Down syndrome or parents of normally developing children. Ollson and Hwang [10] found that mothers of children with autism had higher depression 
scores than mothers of children with intellectual disability. Some research has shown that mothers of children with autism have increased risk of depression $[4,8]$. Furthermore, mothers and fathers of autistic children have differences in mental health status. For example, mothers tend to have higher depression scores than fathers [10].

There have been a few studies on the relationship between parenting stress and characteristics of autism focused on finding the sources of stress. Some studies have indicated that even after intervention, mothers still report their children as a source of stress [11]. Other studies have found that the severity of symptoms and behavior problems are associated with stress $[6,12,13]$.

However, research regarding sources of stress has primarily focused on autistic children's characteristics. The higher level of stress experienced by parents of these children has a negative effect on their physical and psychological status and seriously affects quality of life. However, little attention has been paid to mood changes of parents and the relationship between stress and mood. Therefore, the purposes of this study were to assess stress and mood of parents with autistic children and explore sources of stress and distress as well as the relationship between stress and parents' psychological status (depressive and anxiety symptoms). This information could assist professionals in supporting parents with autistic children and help relieve their psychological burden.

We hypothesized that (i) parents of children with autism would have higher parenting stress scores than parents of normally developing children, (ii) parents of autistic children experience more depressive and anxiety symptoms than parents of normally developing children, and (iii) parents' stress is associated with their own depression and anxiety as well as abnormal behaviors and severity of autism symptoms in their children.

\section{Method}

\subsection{Participants}

\subsubsection{Autism group}

Subjects were 196 parents of children diagnosed with childhood autism using DSM-IV criteria at Shanghai Mental Health Center, Shanghai Jiao Tong University School of Medicine from May 2007 to June 2009. The mean age of fathers was 37.08 years $(\mathrm{SD}=5.91$, range $19-62)$, and mean age of mothers was 34.26 years $(\mathrm{SD}=4.58$, range 25-50). The mean education level of fathers was 13.66 years $(\mathrm{SD}=3.25$, range 6-22), and that of mothers was 12.91 years $(\mathrm{SD}=3.25$, range $6-22)$. There were 170 boys and 26 girls, with a male-female ratio of $6.5: 1$. The age of children ranged from 23 to 144 months (mean age 77.73 months). Parents of the autistic children provided informed consent.

\subsubsection{Control group}

Subjects were parents of normally developing children selected randomly from kindergartens and primary schools. The mean age of fathers was 35.99 years $(\mathrm{SD}=4.17$, range $22-47)$, and mean age of mothers was 34.35 years ( $\mathrm{SD}=4.13$, range 22-45). The mean education level of fathers was 14.74 years ( $\mathrm{SD}=4.12$, range 6-22), and mean education level of mothers was 14.19 years $(\mathrm{SD}=4.30$, range 6-22). There were 89 boys and 24 girls, with a male: female ratio of 3.71:1. The children ranged in age from 23 to 143 months (mean age 77.76 months). Parents provided informed consent.

There were no significant differences observed between the two groups with regard to the age of fathers, age of mothers, child's gender, or age $(P>0.05)$. However, education level of fathers and mothers in the control group was higher than that of parents in the autism group $(P<0.05)$. The data of the two groups were comparable.

\subsection{Measures}

\subsubsection{Parenting stress index (PSI)}

The PSI [14] is a self-report measure designed to evaluate stress in parent-child relationships. It contains 120 items scored on a 5-point Likert scale, from 1 (strongly agree) to 5 (strongly disagree). The PSI assesses two domains of stress: child and parent. The child domain comprises six subscales: adaptability, acceptability, distractability/hyperactivity, demandingness, mood, and reinforces parent. The parent domain has seven subscales: competence, social isolation, attachment, parent health, role restriction, depression, and relationship with spouse. Higher scores indicate greater parenting stress.

\subsubsection{Autism behavior checklist (ABC)}

The ABC, developed by Krug, Arick, and Almond [15], has 57 items containing five categories: sensory, relating, body and object use, language, and social and self-help. In this study, the $\mathrm{ABC}$ was not used as a diagnostic instrument.

\subsubsection{The childhood autism rating scale (CARS)}

The CARS [16] is a 15-item instrument developed to assess parents' perceptions of their children's level of functioning. Items are rated on a 4-point scale ranging from 1 (normal for chronological age) to 4 (severely abnormal). Scores from 30 to 37 indicate light to moderate abnormality. Scores above 37 indicate severe abnormality.

\subsubsection{Self-rating depression scale (SDS)}

The SDS [17] consists of 20 items. Each item is scored from 1 to 4 .

\subsubsection{Self-rating anxiety scale (SAS)}

The SAS [18] consists of 20 items. Each item is scored from 1 to 4 . Level of anxiety was evaluated using this instrument. 


\subsection{Procedure}

All parents of autistic children completed the five scales under supervision of the professional staff. For the normally developing children, parents took the five scales home and sent them to the teacher when they finished. We repaired missing date for the PSI according to the method provided by Abidin [14]. Finally, we input the data and created a database.

\subsection{Statistical analyses}

Data were analyzed with the Statistical Package for the Social Sciences (SPSS) for Windows (version 15.0). We used $t$-tests to explore possible differences on the PSI, ABC, and CARS. Pearson correlations were calculated for the autism group to investigate correlations among the main variables (children's age, behavior problems and severity of impairment, parenting stress, depression, and anxiety). Stepwise regression analysis was used to examine possible predictors of parenting stress.

\section{Results}

\subsection{Group differences in parenting stress}

An independent samples $t$-test conducted to compare mean scores on total parenting stress indicated that parents of children with autism had higher total stress scores than parents of normally developing controls $(t=13.76, P=0.000)$. Parents in the autism group also reported significantly higher scores on the Child Domain, Parent Domain, and all subscales of the PSI than parents in the control group, $(P=0.000$; Table 1).

\subsection{Comparison of PSI scores for autistic children by age}

Autistic children have deficits in language expression, social relations, and learning. Furthermore, they are less adaptable to new environments than normally developing children. Thus, we supposed that level of stress might be different between parents of pre-school age compared to school-age children. Most children enter school after age six in China. Thus, we selected six years old (72 months) as a boundary age to compare stress between these two groups of parents. Results indicated that parents of school-age autistic children reported higher scores on Adaptability, Acceptability, and Reinforces Parent subscales and total Child Domain than parents of pre-school age children, indicating that older autistic children cause greater stress to parents (Table 2).

\subsection{Comparison of parenting stress by sex of child}

Autistic boys and girls may be different in some aspects. For example, boys may be more likely to exhibit hyperactivity or short attention span and aggressive behavior than girls [19]. An independent samples $t$-test indicated that parents of autistic boys reported higher scores for role restriction (mean=24.46, $\mathrm{SD}=4.97)$ than did parents of autistic girls (mean=22.03, $\mathrm{SD}=6.07 ; P=0.027)$. These higher scores indicate that parents experienced the parental role as restricting their freedom and frustrating them in their attempts to maintain their own identity [14] and that autistic boys caused more stress for parents than did autistic girls.

\subsection{Comparison of depression and anxiety symptoms between parents in the two groups}

A comparison of scores for depressive and anxiety symptoms between parents in the two groups revealed that mothers of children with autism had higher SDS scores than fathers of children with autism, who in turn, had higher SDS scores than control parents $(F=17.561, P=0.000)$. Mothers of children with autism had higher SAS scores than fathers

Table 1 Comparison of PSI scores between parents of autistic children and parents of normally developing controls (mean \pm SD)

\begin{tabular}{|c|c|c|c|c|}
\hline PSI & Autism group $(n=188)$ & Control group $(n=114)$ & $t$ & $P$ \\
\hline Child domain & $147.05 \pm 20.61$ & $110.70 \pm 19.52$ & 15.01 & 0.000 \\
\hline Adaptability & $33.65 \pm 6.96$ & $25.74 \pm 5.56$ & 12.56 & 0.000 \\
\hline Ac acceptability & $22.43 \pm 4.51$ & $15.18 \pm 4.50$ & 13.54 & 0.000 \\
\hline Demandingness & $28.36 \pm 5.10$ & $21.46 \pm 4.65$ & 11.78 & 0.000 \\
\hline Mood & $14.88 \pm 3.40$ & $12.24 \pm 3.11$ & 6.74 & 0.000 \\
\hline Distractability/hyperactivity & $31.18 \pm 4.66$ & $25.08 \pm 4.45$ & 11.23 & 0.000 \\
\hline Reinforces parent & $15.31 \pm 3.93$ & $11.33 \pm 3.01$ & 9.90 & 0.000 \\
\hline Parent domain & $163.95 \pm 22.74$ & $135.41 \pm 24.73$ & 10.11 & 0.000 \\
\hline Depression & $26.33 \pm 5.32$ & $21.50 \pm 5.89$ & 7.33 & 0.000 \\
\hline Attachment & $18.71 \pm 3.71$ & $15.75 \pm 3.22$ & 7.32 & 0.000 \\
\hline Role restriction & $24.12 \pm 5.19$ & $18.20 \pm 5.02$ & 9.73 & 0.000 \\
\hline Competence & $38.76 \pm 6.31$ & $31.11 \pm 6.67$ & 9.78 & 0.000 \\
\hline Isolation & $18.17 \pm 4.02$ & $15.39 \pm 3.54$ & 6.12 & 0.000 \\
\hline Spouse & $21.68 \pm 5.01$ & $18.93 \pm 5.19$ & 4.56 & 0.000 \\
\hline Total PSI score & $311.24 \pm 37.95$ & $246.01 \pm 41.27$ & 13.76 & 0.000 \\
\hline
\end{tabular}


Table 2 Comparison of PSI scores for autistic children by age (mean \pm SD)

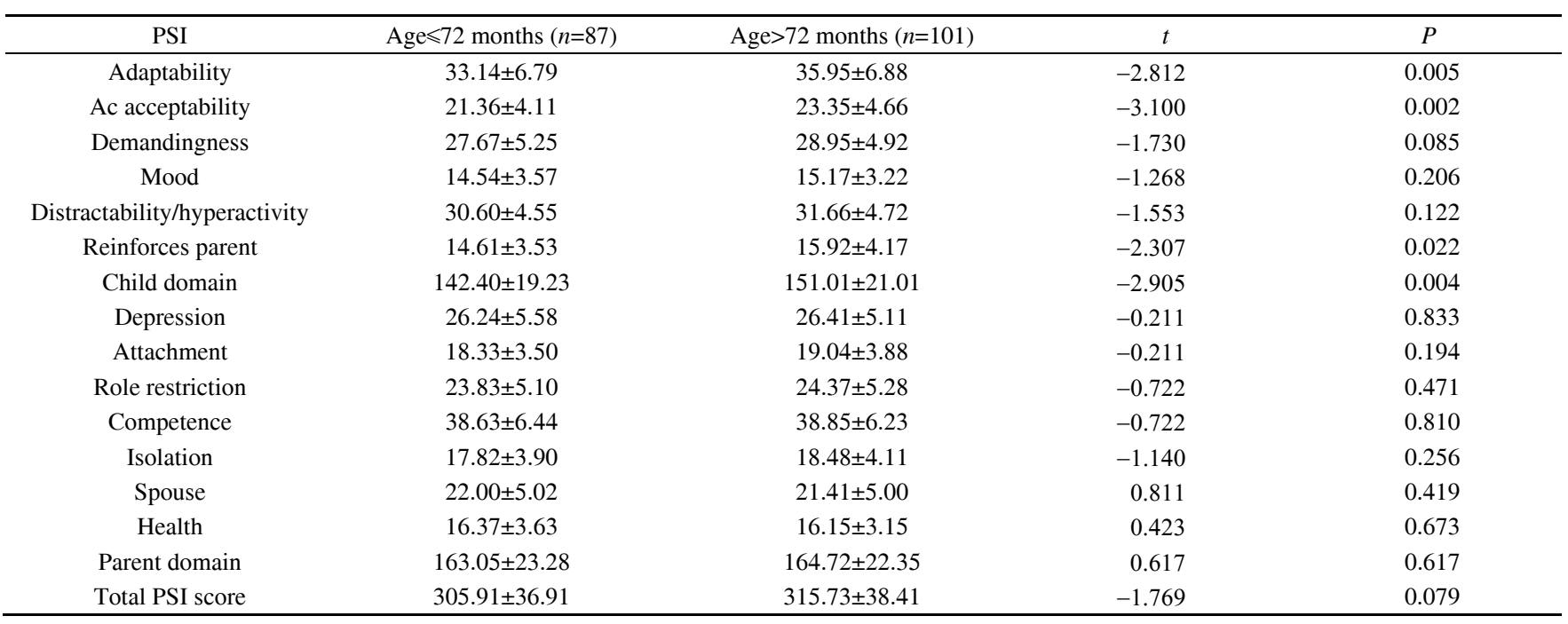

of children with autism, who in turn, had higher SAS scores than control parents $(F=17.535, P=0.000$; Table 3$)$.

We further analyzed percentages of clinical affective symptoms between parents of autistic children and parents in the control group. The SDS scores were significantly higher for mothers with autistic children than for fathers with autistic children and parents in the control group (percentages were $50.3 \%, 31.8 \%$, and $22.7 \%$, respectively). There was no difference between fathers with autistic children and parents in the control group. Elevated symptoms of anxiety were more common in mothers with autistic children $(20.8 \%)$ than that in control parents $(5.1 \%)$. There was no difference between mothers and fathers in the autism group (Table 4).

2.5 Correlative analysis between PSI, ABC, CARS, and affective symptoms among parents with autistic children

Pearson correlation analysis showed that scores for child domain, parent domain, and total PSI were positively related with ABC, CARS scores, and parents' affective symp- toms and ranged from 0.166 to $0.636(P<0.05$ or $P<0.001)$. The age of autistic children was positively associated with child domain scores and total PSI score, but was not associated with parent domain scores. Parent's age and education level and child's gender were not associated with parental stress (Table 5).

\subsection{Related factors predicting parenting stress}

A stepwise linear regression procedure was employed to evaluate the contributions of each variable to parenting stress. Findings indicated that child's age, behavioral problems, and maternal anxiety symptoms were the main predictors of parenting stress, explaining $54.9 \%$ of the variances. The multiple linear regression equation was PSI = $188.765+0.630 X 1+1.249 X 2+0.231 X 3$ (Table 6).

\section{Discussion}

Abidin [14] defined parenting stress as an 'aversive psy-

Table 3 Comparison of SDS and SAS scores between parents with autistic children and parents of normally developing controls

\begin{tabular}{|c|c|c|c|c|c|c|c|c|}
\hline & \multirow{2}{*}{$\begin{array}{c}\text { Fathers } \\
\mathrm{a}(n=170)\end{array}$} & \multirow{2}{*}{$\begin{array}{l}\text { Mothers } \\
\mathrm{b}(n=173)\end{array}$} & \multirow{2}{*}{$\begin{array}{l}\text { Controls } \\
\mathrm{c}(n=75)\end{array}$} & \multirow{2}{*}{$F$} & \multirow{2}{*}{$P$} & \multicolumn{3}{|c|}{$P$} \\
\hline & & & & & & $a / b$ & $\mathrm{a} / \mathrm{c}$ & $\mathrm{b} / \mathrm{c}$ \\
\hline SDS & $45.25 \pm 9.44$ & $49.43 \pm 10.82$ & $41.28 \pm 11.24$ & 17.561 & 0.000 & 0.000 & 0.000 & 0.006 \\
\hline SAS & $38.78 \pm 8.22$ & $41.95 \pm 9.42$ & $36.37 \pm 8.09$ & 12.535 & 0.000 & 0.001 & 0.041 & 0.000 \\
\hline
\end{tabular}

Table 4 Percentage of SDS and SAS scores among parents in the autism group and control group

\begin{tabular}{|c|c|c|c|c|c|c|c|}
\hline & \multirow{2}{*}{$\begin{array}{c}\text { Fathers } \\
\mathrm{a}\end{array}$} & \multirow{2}{*}{$\begin{array}{c}\text { Mothers } \\
\text { b }\end{array}$} & \multirow{2}{*}{$\begin{array}{c}\text { Control } \\
\mathrm{c}\end{array}$} & \multirow[t]{2}{*}{$\chi^{2}$} & \multicolumn{3}{|c|}{$P$} \\
\hline & & & & & $\mathrm{a} / \mathrm{b}$ & $\mathrm{a} / \mathrm{c}$ & $\mathrm{b} / \mathrm{c}$ \\
\hline \multicolumn{8}{|c|}{ SDS score } \\
\hline$\geqslant 50$ & $54(31.8 \%)$ & $87(50.3 \%)$ & $17(22.7 \%)$ & 21.416 & 0.000 & 0.148 & 0.000 \\
\hline \multicolumn{8}{|c|}{ SAS score } \\
\hline$\geqslant 50$ & $23(13.1 \%)$ & $36(20.8 \%)$ & $4(5.1 \%)$ & 11.361 & 0.054 & 0.055 & 0.002 \\
\hline$<50$ & $153(86.9 \%)$ & $137(79.2 \%)$ & $75(94.9 \%)$ & & & & \\
\hline
\end{tabular}


Table 5 Correlations between PSI, CARS, ABC scores, and parental affective symptoms ${ }^{\text {a) }}$

\begin{tabular}{|c|c|c|c|}
\hline & Child domain & Parent domain & Total score of PSI \\
\hline TOTAL CARS & $0.598^{* *}$ & $0.297^{*}$ & $0.505^{* *}$ \\
\hline \multicolumn{4}{|l|}{$\mathrm{ABC}$} \\
\hline Sensory (S) & $0.537^{* *}$ & $0.234^{* *}$ & $0.428^{* *}$ \\
\hline Body and object use (B) & $0.573^{* *}$ & $0.173^{* *}$ & $0.412^{* *}$ \\
\hline Language (L) & $0.438^{* *}$ & $0.250^{* *}$ & $0.374^{* *}$ \\
\hline Social and self-help (S) & $0.579^{* *}$ & $0.298^{* *}$ & $0.490^{* *}$ \\
\hline Total & $0.636^{* *}$ & $0.310^{* *}$ & $0.522^{* *}$ \\
\hline \multicolumn{4}{|l|}{ Maternal affective symptoms } \\
\hline SDS & $0.211^{* *}$ & $0.5400^{* *}$ & $0.435^{* *}$ \\
\hline SAS & $0.166^{*}$ & $0.437^{* *}$ & $0.350^{* *}$ \\
\hline \multicolumn{4}{|l|}{ Paternal affective symptoms } \\
\hline Depression & $0.265^{* *}$ & $0.293^{* *}$ & $0.325^{* *}$ \\
\hline Anxiety & $0.217^{* *}$ & $0.245^{* *}$ & $0.261^{* *}$ \\
\hline Child's age & $0.232^{* *}$ & 0.085 & $0.169^{*}$ \\
\hline
\end{tabular}

a) $*, P<0.05 ; * *, P<0.01$.

Table 6 Stepwise regression analyses predicting parenting stress ${ }^{\text {a) }}$

\begin{tabular}{ccccccc}
\hline & $R^{2}$ & $R$ & $F$ & $P$ & $B$ & SE \\
\hline$X 1$ & $0.391^{\mathrm{a}}$ & 0.625 & 60.981 & $0.000^{\mathrm{a}}$ & 0.630 & 0.624 \\
\hline$X 2$ & $0.503^{\mathrm{b}}$ & 0.709 & 47.493 & $0.000^{\mathrm{b}}$ & 1.249 & 0.316 \\
\hline$X 3$ & $0.549^{\mathrm{c}}$ & 0.741 & 37.706 & $0.000^{\mathrm{b}}$ & 0.231 & 0.216 \\
\hline
\end{tabular}

a) $X 1$, total ABC score; $X 2$, maternal anxiety symptoms; $X 3$, Child's age. a, Predictors: (constant), total ABC score; b, predictors: (constant), total $\mathrm{ABC}$ score, maternal anxiety symptoms; c, predictors: (constant), total ABC score, maternal anxiety symptoms, child's age.

chological reaction to the demands of being a parent'. That researcher regarded parenting stress as related to factors inherent in children, inherent in parents, and in parent-child interaction. Childhood autism is a subtype of pervasive developmental disorders characterized by severe deficits in social interaction and communication, handicaps in verbal and non-verbal communication, an extremely limited range of activities and interests, and stereotypic and repetitive behavior. Most children also have intellectual disability and abnormal feelings. Most families in China have only one child, so parents may expect too much of their child. There is no effective treatment for autism disorder outside of special education. Because the prognosis for children with childhood autism is poor, parents have a high level of stress. Research in the past has indicated that these parents have higher stress than parents of children with Down syndrome or parents of normally developing children $[4,8]$.

This study showed that parents with autistic children have a higher level of stress than parents of normally developing children based on higher scores for child domain and parent domain subscales as well as total PSI score, consistent with research by Hoffman and Baker-Ericzén [6,20]. Means for the child domain subscale were at the 99th percentile, according to norms reported by Abidin [14], indicating that demands of children, behavior problems difficult to manage, and unstable feelings cause great stress for parents. Parent domain scores were also very high, with means at approximately the 95th percentile. Mean scores for all parent domain subscales, except relationship with spouse, were above the 85th percentile, indicating that less emotional closeness, feelings of isolation, and a lack of help may also be stressful to parents.

Abidin pointed out that stress is high if the total PSI score is at or above 260, and parents should be referred for professional consultation [14]. Our findings showed that $91.9 \%$ of parents of children with autism reported elevated total PSI scores, which was significantly higher than for the control group (42.6\%). This indicates that parents of children with autism were more vulnerable to elevated stress. A study on Asperger's syndrome by Epstein [21] reported that $75.7 \%$ of mothers and $75 \%$ of fathers reported high stress levels. Baker-Ericzén [20] reported that 59\% of mothers with ASD children reported higher levels of stress on the child domain of the PSI. Tomanik et al. [22] showed that two out of three mothers of children with autism reported higher levels of stress on the child domain subscale. Lecavalier [23] reported that $57.7 \%$ of parents of children with ASD scored in the clinically significant range. The percentage of parents in our sample above the PSI clinical cut-point was higher than that reported by other researchers, possibly because medical care systems in other countries are better than in China, and there are more professionals providing systematic and professional training than in our country. In addition, the expensive cost of training in China may increase the economic burden of parents with autistic children. Therefore, only one child suffering from autism in a family may increase mental health issues for parents.

Our results showed that parents of school age autistic children reported higher levels of stress on the adaptability, acceptability, and reinforces parent subscales and total child domain of the PSI than parents of pre-school age autistic children. This finding is similar to previous research reporting higher stress in mothers of older compared to younger adolescents [24].

Because autistic children have deficits in language expression, social interaction, and learning, when they enter 
school there may be difficulties in learning and interaction with peers, and it may be difficult for them to adapt to the school environment. Correlation analyses in this study showed a positive relation between child's age and scores for the child domain subscale and total PSI, but no association of age with parent domain scores. Results indicated that as the children grow, their behavioral problems can increase parental stress. However, results of Duarte et al. [25] were contrary to ours. Other studies have found that age is not associated with parenting stress [23,26]. Evidence is mixed regarding the effect of age on parenting stress, with a probable reason being the different age ranges studied.

Our study found that parents of autistic boys had higher role restriction scores than parents of autistic girls. These higher scores indicate that being a parent restricts parental freedom, and parents feel they are controlled by the demands and needs of their children [14]. This result suggests that parenting a boy with autism may be more stressful to parents than parenting a daughter with this disorder. Indeed, one study reported that parenting a boy with autism was more stressful for mothers, possibly because of the size and strength of boys as they grow up [27]. Some research has indicated that mothers of a daughter with ASD have greater parenting stress than mothers with an autistic son [28]. However, other studies have found that gender is not associated with parental stress [6,23,29]. Findings regarding effect of gender on parenting stress should be viewed cautiously because correlation analyses in this study showed no significant correlation between child's gender and PSI scores. Given that there is little research on the effect of gender on parenting stress, future studies might focus on this aspect.

Both fathers and mothers in the autism group reported significantly higher depression and higher anxiety scores than control parents, consistent with research by Hamlyn-Wright [9]. Other researchers have found that mothers of children with autism had higher depression scores than mothers of normally developing children, mothers of children with intellectual disability, and Down syndrome $[7,8,10]$. In a comparison of the mean score for fathers with that for mothers of children with autism, we found that mothers had higher depressive and anxiety scores than fathers. Some previous studies have reported the same result $[10,30]$. However, one study reported no difference in level of depression between fathers and mothers, but mothers indeed reported more anxiety symptoms than fathers [31].

In the present study, over half of mothers of children with autism had higher levels of depressive feelings, and one-fifth experienced higher anxiety symptoms than mothers in the control group. More than one-third of fathers of children with autism reported elevated depressive symptoms, and about $13 \%$ of these fathers reported an elevated level of anxiety. Results suggest that mothers of children with autism are more likely to experience elevated levels of depression than fathers, consistent with previous research [32].
Ollson and Hwang [10] and Bailey et al. [33] found that mothers of children with autism are at an increased risk of depression. The review by Bailey et al. [33] reported that four studies indicated rates of lifetime history of depression rates as $10 \%, 27 \%, 33 \%$, and $59 \%$ for mothers of children with autism. Although our study indicated that fathers of an autistic child had higher depression scores than controls, there was no significant difference in percentage of elevated depression scores between fathers with autistic children and controls, contrary to results reported by Ollson and Hwang [10]. Elevated anxiety scores were more common in mothers of children with autism than in parents of normally developing children, indicating that those mothers were at increased risk of anxiety. There was no difference in percentage of elevated anxiety scores between mothers and fathers with autistic children, consistent with findings by Davis and Carter [32].

In general, most studies indicate that mothers of children with autism have higher levels of depression than fathers, suggesting that they experience more distress than their husbands. A possible explanation for this may be that mothers take on a large part of the extra care and practical work that the child requires. In addition, mothers are more likely to give up their jobs and may be unable to pursue their own interests [10].

Correlation analysis showed that affective symptoms of mothers and fathers of autistic children were negatively related to their level of education and that of their spouse, suggesting that parents with less education feel more distressed. They may be less likely to get comfort from discussions with their spouses, and they may not know how to get help from other resources when they face difficulties. Affective symptoms of mothers and fathers were negatively related to family income, indicating that economic problems increase distress in parents of autistic children. Correlation analysis also found that CARS scores were positively associated with depressive symptoms of mothers and fathers and anxiety symptoms in fathers. However, ABC scores were only correlated with anxiety symptoms in fathers. These results suggest that impairment of a child's functioning increases parental distress.

Some research has shown that behavior problems of autistic children are related to mothers' affective symptoms $[21,30,34]$, while pressure is associated with parents' depressive and anxiety symptoms [22,29].

The CARS was used to assess parents' perception of their children's level of functioning. This study showed that the total CARS score was positively related to child domain, parent domain, and total PSI scores. We found that parents of children with severe symptoms had higher total PSI scores than parents of children with light to moderate symptoms, indicating that the more serious the symptoms were, the higher stress perceived by parents.

Total ABC scores were positively related to child domain, parent domain, and total PSI scores, suggesting that the 
more serious the child's behavior problems, the higher the stress perceived by parents. Maternal and paternal affective symptoms were positively associated with total PSI score. This is consistent with research by Orsmod et al. [28] and Davis and Carter [32].

Age was also a factor related to parenting stress, with parents of older autistic children reporting more stress than parents of younger autistic children. As autistic children grow older, their abnormal behavior, deficits in social interaction, and poor self-care may become more obvious. Thus, parents begin to worry about their child's future.

Correlation analyses showed that age, behavior problems, functioning level of autistic children, and parental affective symptoms were associated with parenting stress. Stepwise regression analyses showed that the child's age, behavior problems, and level of maternal anxiety were the main predictors of parenting stress. Children's behavior problems entered the regression equation first and accounted for $39.1 \%$ of the total variation. A child's behavior problems are reportedly the main factor related to maternal stress [31]. In addition, externalized behaviors are strongly associated with parental stress [23]. Brobst et al. [13] found that behavior problems of children with autism spectrum disorder were significantly related to parental stress, and intensity of behavior problems and severity of disability, not number of behavior problems, were most related to stress. There have been other studies reporting that behavior problems of children with autism are related to parenting stress [22]. Given that behavior problems of autistic children are the most important predictor of parenting stress, doctors might reduce that stress by addressing these behavior problems.

The ABC was used to assess children's behavior, and the CARS was used to assess children's level of functioning. Regression analyses in this study showed that children's behavior problems represented the main factor related to parenting stress. Although there was a correlation between children's social functioning and parenting stress, the CARS did not enter the regression equation, suggesting that children's functioning was not the most important factor. Other researchers have found that children's behavior problems, not other factors such as adaptive function, are associated with parental stress [23,31,34]. Tobing and Glenwick [35] found no correlation between CARS score and total PSI score of parents of children with autism. However, there was a trend toward correlation of CARS score and Parent Domain score, with this researcher concluding there might be a correlation if the sample size was enlarged. A study by Phetrasuwan and Shandor [29] reported similar results. Those researchers considered the possible reasons why the CARS did not reflect more complicated behavior problems of children with autism spectrum disorder or the children's degree of dependence on mothers [29]. Davis and Carter [32] revealed that externalized behaviors of children were associated with paternal stress, and dysregulation of sleeping and eating were associated with maternal stress. However, cognitive functioning, communication deficits, and atypical behavior were not associated with parenting stress.

From the discussion above, we can conclude that behavior problems are the main factor associated with parental stress. However, there are inconsistent conclusions regarding the contribution of children's functioning on such stress. This may be because different assessments have been used to evaluate stress, behavior problems, and children's functioning. Future studies should select instruments with good psychometric properties to disentangle these relationships.

In this study, the three variables entered into the regression equation only accounted for $54.9 \%$ of the total variation, indicating there were other factors related to parenting stress. For example, some research has shown that parents' personal characteristics, social support, and coping styles are related to perceived stress. Future studies should take into account these factors.

There are several limitations in this study. First, we did not measure mothers' and fathers' stress independently. Therefore, results do not reflect separately reported stress levels of parents. Second, we used the SDS and SAS to measure parental affective symptoms, which only reflect present and not past symptoms or predict future mood. Although there were limitations in this study, our sample size was large. We believe our findings can play an important role in clinical instruction regarding stress in parents of autistic children.

We are grateful to all the children and parents who participated in this study. This work was supported by the National Basic Research Program of China (Grant No. 2010CB529602).

1 Schieve LA, Blumberg SJ, Rice C, Visser SN, Boyle C. The relationship between autism and parenting stress. Pediatrics, 2007, 119: S114-121

2 Fombonne E. Epidemiology of autistic disorder and other pervasive developmental disorders. J Clin Psychiatry, 2005, 66: 3-8

3 Zaidman-Zait A, Mirenda P, Zumbo BD. An item response theory analysis of the Parenting Stress Index-Short Form with parents of children with autism spectrum disorders. J Child Psychol Psychiatry, 2010, 51: 1269-1277

4 Wolf L C, Noh S, Fisman SN, Speechley M. Psychological effects of parenting stress on parents of autistic children. J Autism Dev Disord, 1989, 19: 157-166

5 Baker BL. Blacher J, Crnic KA. Behavior problems and parenting stress in families of three-year-old children with and without developmental delays. Am J Ment Retard, 2002, 107: 433-444

6 Hoffman CD, Sweeney DP, Hodge D. Parenting stress and closeness: mothers of typically developing children and mothers of children with autism. FOCUS, 2009, 24: 178-187

7 Abbeduto L, Seltzer MM, Shattuck P, Krauss MW, Orsmond G, Murphy MM. Psychological well being and coping in mothers of youths with autism, Down syndrome, or fragile X syndrome. Am J Ment Retard, 2004, 109: 237-254

8 Dumas JE, Wolf LC, Fishman SN, Culligan, A. Parenting stress, child behavior problems, and dysphoria in parents of children with autism, Down syndrome, behavior disorders, and normal development. Exceptionality, 1991, 2: 97-110

9 Hamlyn-Wright S, Draghi-Lorenz R, Ellis J. Locus of control fails to mediate between stress and anxiety and depression in parents of chil- 
dren with a developmental disorder. Autism, 2007, 11: 489-501

10 Olsson MB, Hwang CP. Depression in mothers and fathers of children with intellectual disability. J Intellect Disabil Res, 2001, 45: 535-543

11 Boyd BA. Examining the relationship between stress and lack of social support in mothers of children with autism. FOCUS, 2002, 17: 208-216

12 Hastings RP. Child behaviour problems and partner mental health as correlates of stress in mothers and fathers of children with autism. J Intellect Disabil Res, 2003, 47: 231-237

13 Brobst JB, Clopton JR, Hendrick SS. Parenting children with autism spectrum disorders: the couple's relationship. FOCUS, 2009, 24: 38-49

14 Abidin RR. Parenting Stress Index. 3rd ed. Odessa, FL: Psychological Assessment Resource, 1995

15 Krug DA, Arick JR, AlmondP. Behavior checklist for identifying severely handicapped individuals with high levels of autistic behavior. J Child Psychol Psychiatry, 1980, 21: 221-229

16 Schopler E, Reichler RJ, DeVellis RF. Toward objective classification of childhood autism: childhood autism rating scale (CARS). J Autism Dev Disord, 1980, 10: 91-103

17 Zung WW. A self-rating depression scale. Arch Gen Psychiatry, 1965, 12: 63-70

18 Zung WW. A rating instrument for anxiety disorders. Psychosomatics, 1971, 12: 371-379

19 Giarelli E, Wiggins LD, Rice CE, Levy SE, Kirby RS, Pinto-Martin J, Mandell D. Sex differences in the evaluation and diagnosis of autism spectrum disorders among children. Disabil Health J, 2010, 3: 107-116

20 Baker-Ericén MJ, Brookman-Frazee L, Stahmer A. Stress levels and adaptability in parents of toddlers with and without autism spectrum disorders. Res Pract Persons Severe Disabil, 2005, 30: 194-204

21 Epstein T, Saltzman-Benaiah J, O'Hare A, Goll JC, Tuck S. Associated features of Asperger syndrome and their relationship to parenting stress. Child Care Health Dev, 2008, 34: 503-511

22 Tomanik S, Harris GE, Hawkins J. The relation-ship between behaviours exhibited by children with autism and maternal stress. J Intellect Devel Disabil, 2004, 29: 16-26
23 Lecavalier L, Leone S, Wiltz J. The impact of behaviour problems on caregiver stress in young people with autism spectrum disorders. J Intellect Disabil Res, 2006, 50: 172-183

24 Donovon AM. Family stress and ways of coping with adolescents who have handicaps: maternal perceptions. Am J Ment Retard, 1988, 92: 502-509

25 Duarte CS, Bordin IA, Yazigi L. Factors associated with stress in mothers of children with autism. Autism, 2005, 9: 416-427

26 Koegel RL, Schreibman L, O'Neill RE, Burke JC. The personality and family-interaction characteristics of parents of autistic children. $\mathrm{J}$ Consult Clin Psychol, 1983, 51: 683-692

27 Bristol MM, Gallagher JJ, Schopler E. Mothers and fathers of young developmentally disabled and non-disabled boys: adaption and spouse support. Dev Psychol, 1988, 24: 441-451

28 Orsmod GI, Lin LY, Seltzer MM. Mothers of adolescents and adults with autism: parenting multiple children with disabilities. Intellect Dev Disabil, 2007, 45: 257-270

29 Phetrasuwan S, Shandor MM. Parenting stress in mothers of children with autism spectrum disorders. J Spec Pediatr Nurs, 2009, 14: 157-165

30 Hastings RP, Kovshoff H, Ward NJ, degli Espinosa F, Brown T, Remington B. System analysis of stress and positive perceptions in mothers and fathers of pre-school children with autism. J Autism Dev Disord, 2005, 35: 635-644

31 Hastings RP. Child behaviour problems and partner mental health as correlates of stress in mothers and fathers of children with autism. J Intellect Disabil Res, 2003, 47: 231-237

32 Davis NO, Carter AS. Parenting stress in mothers and fathers of toddlers with autism spectrum disorders: Associations with child characteristics. J Autism Dev Disord, 2008, 38: 1278-1291

33 Bailey DBJr, Golden RN, Roberts J, Ford A. Maternal depression and developmental disability: research critique. Ment Retard Dev Disabil Res Rev, 2007, 13: 321-329

34 Estes A, Munson J, Dawson G. Parenting stress and psychological functioning among mothers of preschool children with autism and developmental delay. Autism, 2009, 13: 375-387

35 Tobing LE, Glenwick DS. Relation of the childhood autism rating scale-parent version to diagnosis, stress, and age. Res Dev Disabil, 2002, 23: 211-223

Open Access This article is distributed under the terms of the Creative Commons Attribution License which permits any use, distribution, and reproduction in any medium, provided the original author(s) and source are credited. 